(C) 2016 IEEE. Personal use of this material is permitted. Permission from IEEE must be obtained for all other uses, in any current or future media, including reprinting/republishing this material for advertising or promotional purposes, creating new collective works, for resale or redistribution to servers or lists, or reuse of any copyrighted component of this work in other works. 


\title{
Fast Multistep Finite Control Set Model Predictive Control for Transient Operation of Power Converters
}

\author{
Roky Baidya*, Ricardo P. Aguilera ${ }^{\dagger}$, Pablo Acuna*, Ramon Delgado ${ }^{\ddagger}$, Tobias Geyer ${ }^{\S}$, \\ Daniel Quevedo", Toit Mouton" \\ *Australian Energy Research Institute, University of New South Wales, Australia, \\ $\dagger$ The School of Electrical, Mechanical and Mechatronic Systems, The University of Technology Sydney (UTS), Australia, \\ ${ }^{\ddagger}$ School of Electrical Engineering, The University of Newcastle, Australia, \\ $\S$ ABB Corporate Research, Switzerland, \\ IDepartment of Electrical Engineering (EIM-E), Paderborn University, Germany. \\ "Department of Electrical and Electronic Engineering, University of Stellenbosch, South Africa. \\ Email: raguilera@ieee.org
}

\begin{abstract}
Recently, an efficient optimization strategy based on the sphere decoding algorithm (SDA) has been proposed to solve the optimal control problem underlying direct model predictive control (MPC) formulations with long horizons. However, as will be elucidated in this work, this optimization algorithm presents some limitations during transient operation of power converters, which increase the execution time required to obtain the optimal solution. To overcome this issue, the present work presents an improved version of the SDA for direct MPC that is not affected by transient operations of the power converter. The key novelty of the proposal is to reduce the execution time of the SDA when the system is in a transient by projecting the unconstrained optimal solution onto the envelope of the original finite control set. As evidenced by the simulation results, the proposed SDA is able to quickly compute the optimal solution for the long-horizon direct MPC during both steady-state and transient operation of the power converter.
\end{abstract}

Index Terms- Finite control set, predictive control, multilevel converters, optimization problem, sphere decoding, convex hull.

\section{INTRODUCTION}

Multilevel converters (MCs) are the preferred technology for medium/high-voltage and high power applications [1], [2]. Several MC topologies have been proposed in the literature, which offer different features mainly related with high-quality voltage and currents waveforms at medium/high-voltage using low voltage rated semiconductor switches. Traditional modulation techniques or special methods can be used to provide a multilevel output voltage with improved power quality (i.e., low harmonic profile, low $d v / d t$ ratings, etc). Thus, MCs are of special importance in high power applications, such as highpower drives [3], active power filters [4], and renewable energy applications [5], [6], among others.

Model predictive control (MPC) for MCs has become an interesting option due to its attractive features e.g., fast dynamic response, easy inclusion of nonlinearities and constraints, etc [7]. Several works can be found in the literature utilizing the MPC successfully for MCs, e.g., [8]-[13]. Among the predictive control families, the so-called Finite-Control-Set MPC (FCS-MPC) or direct MPC is the most popular one for power converters [14], [15]. FCS-MPC directly considers the switching states or voltage levels as control inputs into the optimization problem. In the standard approach, the optimal control input is found by evaluating a finite set of input combinations in a cost function which has minimum cost value. Therefore, a modulation stage is not required.

In recent works, the importance of using long horizon direct MPC schemes has been demonstrated [16], [17]. The advantage of moving to longer horizons is to obtain an improved steady-state performance in terms of harmonic distortion and switch commutations. However, the required computational effort is challenging since the number of input combinations increases exponentially with the horizon length. This issue becomes a particular concern when implementing the controllers standard control platform such as FPGAs, DSPs, etc [18], [19]. Recent research outcomes show that the computational burden can be reduced significantly by introducing an efficient optimization strategy named Sphere Decoding Algorithm (SDA) [20]-[22]. Nevertheless, as will be elucidated in this work, standard SDA presents some limitations during transient operations of power converters, which increase the execution time required to obtain the optimal solution. This compromises the implementation of SDA to solve the associated optimal problem of FCS-MPC governing power converters.

The work at hand presents an improved version of the SDA, which tackles the aforementioned issues. Here, the controller is directly formulated in the original $a b c$-framework. The key novelty of the proposal is to quickening the SDA, when the system is in transient by projecting the unconstrained optimal solution on the envelope of the original finite control set. As evidenced by the simulation results, the proposed SDA is able to quickly compute the optimal solution for the long-horizon direct MPC during both steady-state and transient operations of the power converter. 


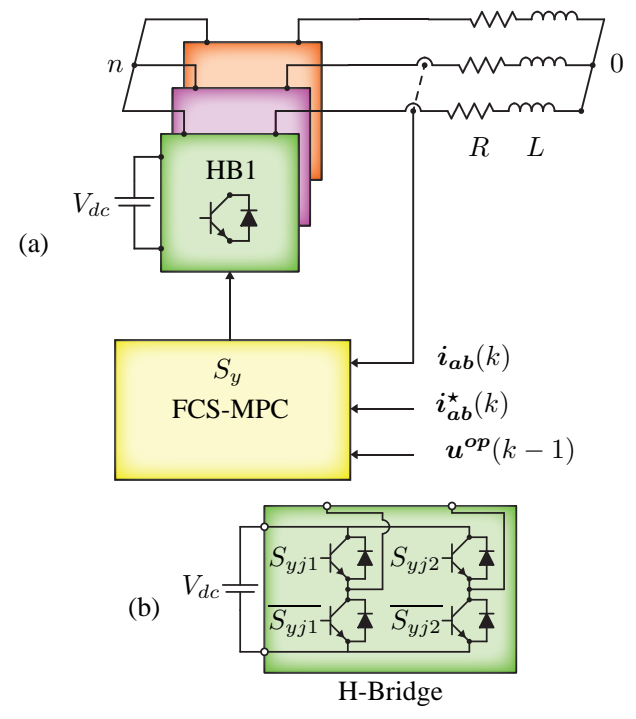

Fig. 1. Schematic of a three-phase three-level HB inverter: (a) block diagram of the FCS-MPC; (b) single H-Bridge converter

\section{FCS-MPC FORMULATION}

To employ the new proposal of interest, this paper considers the use of FCS-MPC to govern a three-level three-phase $\mathrm{H}$ Bridge (HB) inverter shown in Fig. 1. Here, each HB cell is electrically fed with an isolated dc voltage source. The inverter supply power to a passive RL load. This section introduces the system model and the optimization problem.

\section{A. Converter Model}

From the basic circuit analysis, we can express the continuous-time dynamic model of the inverter as:

$$
\frac{d i_{y}(t)}{d t}=-\frac{R}{L} i_{y}(t)+\frac{1}{L}\left(v_{y n}(t)-v_{0 n}(t)\right)
$$

for all $y \in\{a, b, c\}$ and $v_{y n}(t)$ is the individual cell output voltage. The common mode voltage $\left(\mathrm{CMV}, v_{0 n}\right)$ is given by:

$$
v_{0 n}(t)=\frac{1}{3}\left(v_{a}(t)+v_{b}(t)+v_{c}(t)\right) .
$$

Usually in power electronics, the power switches are considered as control input [23]. This may lead to a large number of input combinations, which may difficult unnecessarily the implementation of FCS-MPC. To account for this, this work considers the phase voltage levels, $v_{\ell y}$, as control input [22]. Thus, for a generic $\eta$-cells converter, the output voltage becomes:

$$
v_{y n}(t)=\frac{V_{d c}}{\eta} v_{\ell y}(t)
$$

where $\eta$ is the number of cell per phase ( $\eta=1$ for one-cell) in the converter and,

$$
v_{\ell y}(t) \in \mathbb{V} \triangleq\{-\eta,-\eta+1, \ldots, 0, \ldots, \eta-1, \eta\} .
$$

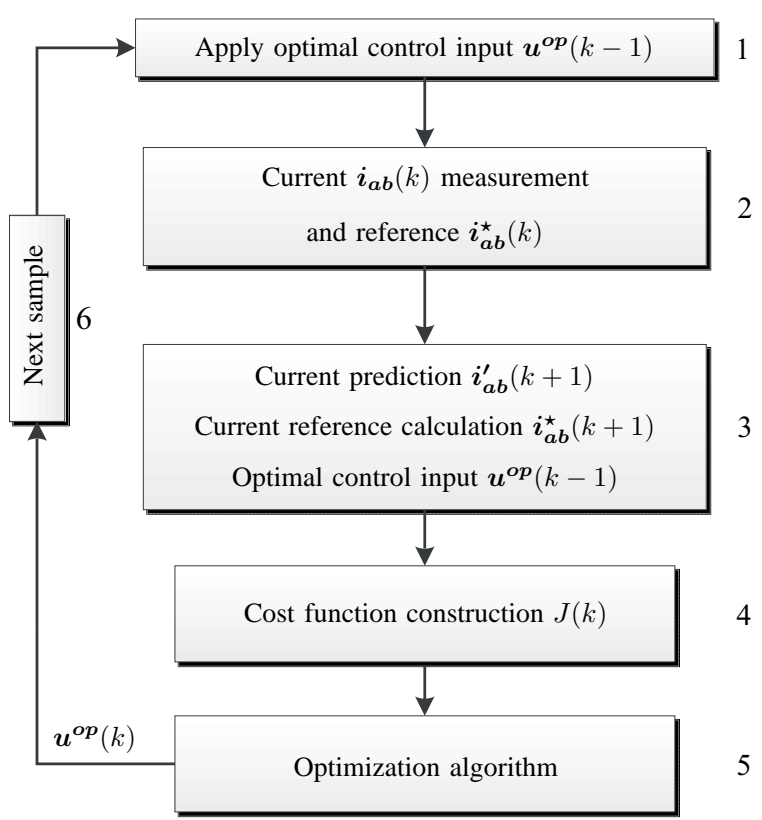

Fig. 2. Flow diagram of the proposed FCS-MPC strategy for horizon one

Here, we implement the FCS-MPC for a HB inverter in the original $a b c$-framework. The system states and control inputs are chosen as:

$$
\boldsymbol{i}_{\boldsymbol{a} \boldsymbol{b}}(k)=\left[\begin{array}{c}
i_{a}(k) \\
i_{b}(k)
\end{array}\right] \in \mathbb{R}^{2}, \quad \boldsymbol{u}(k)=\left[\begin{array}{l}
v_{\ell a}(k) \\
v_{\ell b}(k) \\
v_{\ell c}(k)
\end{array}\right] \in \mathbb{U},
$$

where $i_{c}(k)=-\left(i_{a}(k)+i_{b}(k)\right)$ and the input, $\boldsymbol{u}(k)$, belongs to the finite control set $\mathbb{U} \triangleq \mathbb{V}^{3}$. Now, the discrete-time dynamic model can be obtained by applying the forward Euler discretization to (1) with a sampling period of $T_{s}$, i.e.,:

$$
\boldsymbol{i}_{\boldsymbol{a b}}(k+1)=\boldsymbol{A} \boldsymbol{i}_{\boldsymbol{a b}}(k)+\boldsymbol{B} \boldsymbol{u}(k),
$$

where

$$
\boldsymbol{A}=\left[\begin{array}{cc}
1-\frac{R T_{s}}{L} & 0 \\
0 & 1-\frac{R T_{s}}{L}
\end{array}\right], \boldsymbol{B}=\frac{V_{d c} T_{s}}{3 L}\left[\begin{array}{ccc}
2 & -1 & -1 \\
-1 & 2 & -1
\end{array}\right]
$$

\section{B. Optimal Control Problem}

In this work, our control target focuses on the reference current tracking and the switching effort at each time step- $k$. The first target is to track the current reference, $i_{a b}^{\star}$, for the HB output current, i.e.,:

$$
i_{a b}^{\star}(t)=\left[\begin{array}{c}
I^{\star} \sin (\omega t) \\
I^{\star} \sin (\omega t-2 \pi / 3)
\end{array}\right],
$$

where, $I^{\star}$ is the amplitude of the current references.

The second target is the switching effort $\Delta \boldsymbol{u}^{\prime}(k)=\boldsymbol{u}^{\prime}(k)-$ $\boldsymbol{u}^{\boldsymbol{o p}}(k-1)$. Here, $\boldsymbol{u}^{\boldsymbol{o p}}(k-1)$ is the optimal solution found at the last step $(k-1)$. Thus, the cost function penalizes the current tracking error and the switching effort as shown below for the horizon length $N=1$,

$$
J(k)=\left\|\boldsymbol{i}_{\boldsymbol{a} \boldsymbol{b}}^{\prime}(k+1)-\boldsymbol{i}_{\boldsymbol{a} \boldsymbol{b}}^{\star}(k+1)\right\|_{2}^{2}+\lambda\left\|\Delta \boldsymbol{u}^{\prime}(k)\right\|_{2}^{2} .
$$


Here, $\boldsymbol{i}_{\boldsymbol{a} \boldsymbol{b}}^{\prime}(k+1)$ stands for the tentative HB current predictions that is generated by the tentative control input $\boldsymbol{u}^{\prime}(k)$. In (9), $\|\cdot\|_{2}^{2}$ represents the quadratic eucledian norm, i.e., $\left\|\boldsymbol{a}-\boldsymbol{a}^{\star}\right\|_{2}^{2}=\left(a_{1}-a_{1}^{\star}\right)^{2}+\ldots+\left(a_{p}-a_{p}^{\star}\right)^{2}$, for a pair of vectors $a, a^{\star} \in \mathbb{R}^{p}$. Additionally, the weighting factor $\lambda$ penalizes the impact on commutations to achieve the desired switching frequency. In recent research work [20], this cost function (9) has been proposed for three-level neutral point clamped voltage source inverter in the the $\alpha \beta$-framework.

The optimal control input $\boldsymbol{u}^{\boldsymbol{o p}}(k)$, which is to be applied by the converter, is the one that minimizes the cost function. Thus, the optimization problem underlying in FCS-MPC strategy can be written as:

$$
\boldsymbol{u}^{\boldsymbol{o p}}(k)=\arg \left\{\min _{\boldsymbol{u} \in \mathbb{U}} J(k)\right\} .
$$

Normally, in power electronics, one step voltage level changes are recommended to avoid high $d v / d t$ rating in the system. Thus, the constraint on the optimal control input to be obtained $\boldsymbol{u}^{\boldsymbol{o p}}(k)$ can be defined as follows:

$$
\left\|\Delta \boldsymbol{u}^{\prime}(k)\right\|_{\infty} \leq 1
$$

A flow diagram of the FCS-MPC strategy for $N=1$ is shown in Fig. 2. This strategy considers the aforementioned cost function (9) by performing in general curent prediction $\boldsymbol{i}_{\boldsymbol{a} \boldsymbol{b}}^{\prime}(k+1)$, current reference calculation $\boldsymbol{i}_{\boldsymbol{a} \boldsymbol{b}}^{\star}(k+1)$ and the optimal control input $\boldsymbol{u}^{\boldsymbol{o p}}(k-1)$ found at previous time step$(k-1)$. The optimal input $\boldsymbol{u}^{\boldsymbol{o p}}(k)$ is obtained by solving (10).

Now, the cost function and the optimization problem for arbitrary horizon length $N$ is:

$$
\begin{gathered}
J_{N}=\sum_{l=k}^{k+N-1}\left\|\boldsymbol{i}_{\boldsymbol{a} \boldsymbol{b}}^{\prime}(l+1)-\boldsymbol{i}_{\boldsymbol{a} \boldsymbol{b}}^{\star}(l+1)\right\|_{2}^{2}+\lambda\left\|\Delta \boldsymbol{u}^{\prime}(l)\right\|_{2}^{2}, \\
\boldsymbol{U}^{\boldsymbol{o p t}}(k)=\arg \left\{\min _{\boldsymbol{U} \in \mathbb{U}} J_{N}\right\} .
\end{gathered}
$$

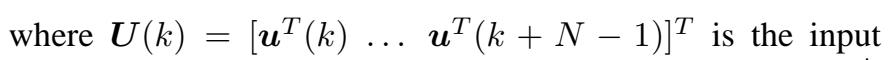
sequences which belongs to the finite input sequence $\mathbb{U} \triangleq$ $\mathbb{V}^{3 N}$, and $\boldsymbol{U}^{\boldsymbol{o p t}}$ is the optimal solution. Interested readers are referred to [23] for a detailed analysis of long horizon FCSMPC.

\section{STANDARD SDA}

This work uses the well known SDA to find the optimal control input, $\boldsymbol{u}^{\boldsymbol{o p}}(k)$, to be applied to the inverter. The importance of SDA for long prediction horizons in power electronics has been shown in the recent research works [20][22]. Here, we briefly discuss the working principle of SDA for the horizon-one case, which helps the reader to understand our proposal.

SDA solves the optimization problem of interest (10) in an equivalent triangular-least squares form. To do this, we rewrite (10) as the following integer quadratic programming problem:

$$
\boldsymbol{u}^{\boldsymbol{o p}}(k)=\arg \min _{\boldsymbol{u} \in \mathbb{U}}\left\{\boldsymbol{u}^{\boldsymbol{T}}(k) \boldsymbol{W} \boldsymbol{u}(k)+2 \boldsymbol{u}^{\boldsymbol{T}}(k) \boldsymbol{F}(k)\right\} .
$$

where

$$
\boldsymbol{F}(k)=\boldsymbol{B}^{\boldsymbol{T}} \boldsymbol{A} \boldsymbol{i}_{\boldsymbol{a} \boldsymbol{b}}(k)-\boldsymbol{B}^{\boldsymbol{T}} \boldsymbol{i}_{\boldsymbol{a} \boldsymbol{b}}^{\star}(k)-\lambda \boldsymbol{u}^{\boldsymbol{o p}}(k-1),
$$

and $\boldsymbol{W}=\boldsymbol{B}^{T} \boldsymbol{B}+\lambda \boldsymbol{I}_{3 \times 3}$ is a symmetric and positive definite matrix for $\lambda \geq 0$. Thus, we can apply the, so-called, Cholesky decomposition [20], [24] on $\boldsymbol{W}$ to find a unique invertible and triangular matrix $\boldsymbol{H} \in \mathbb{R}^{3 \times 3}$, which satisfies:

$$
\boldsymbol{W}=\boldsymbol{H}^{T} \boldsymbol{H} .
$$

When there are no constraints on the input, i.e., $\boldsymbol{u}(k) \in \mathbb{R}^{3}$, the unconstrained optimal solution $\boldsymbol{u}_{\boldsymbol{u c}}^{\text {op }}(k)$ can be expressed in the following manner:

$$
\begin{aligned}
\boldsymbol{u}_{\boldsymbol{u c}}^{\boldsymbol{o p}}(k) & =\arg \min _{\boldsymbol{u} \in \mathbb{R}^{3}}\left\{\boldsymbol{u}^{\boldsymbol{T}}(k) \boldsymbol{W} \boldsymbol{u}(k)+2 \boldsymbol{u}^{\boldsymbol{T}}(k) \boldsymbol{F}(k)\right\} \\
& =-\boldsymbol{W}^{-1} \boldsymbol{F}(k) .
\end{aligned}
$$

Now, using (16) and (17), we can rewrite the integer quadratic problem (14) as:

$$
\boldsymbol{u}^{\boldsymbol{o p}}(k)=\arg \left\{\min _{\boldsymbol{u} \in \mathbb{U}}\left\|\boldsymbol{H} \boldsymbol{u}(k)-\boldsymbol{y}_{\boldsymbol{c}}(k)\right\|_{2}^{2}\right\},
$$

where $\boldsymbol{y}_{\boldsymbol{c}}(k)=\boldsymbol{H} \boldsymbol{u}_{\boldsymbol{u c}}^{\boldsymbol{o p}}(\boldsymbol{k})$.

Notice that (18) is a least-squares quantization problem in triangular form, which is the requirement to implement SDA. It is important to highlight that obtaining the optimal solution $\boldsymbol{u}^{\boldsymbol{o p}}(k)$ by solving the minimization in (10) is equivalent to the quantization as per (18).

The SDA solves the above quantization problem (18) by searching the tentative solutions inside the sphere. This sphere is defined with the center $\boldsymbol{y}_{c}$ and an initial radius $\boldsymbol{\rho}$, as proposed in [20]. To concise the paper we discard the initial radius selection method. Interested readers are referred to [20] for detail analysis.

A flow diagram of the SDA for three-phase three-level HB inverter is shown in Fig. 4. Taking three precalculated parameters $\left(\boldsymbol{H}, \boldsymbol{u}_{\boldsymbol{u c}}^{\boldsymbol{o p}}\right.$ and $\left.\rho^{2}\right)$, the algorithm starts searching the prospective solution in a repetitive manner, which satisfy the following condition:

$$
\left\|\boldsymbol{H} \boldsymbol{u}(k)-\boldsymbol{y}_{\boldsymbol{c}}(k)\right\|_{2}^{2} \leqslant \rho(k) .
$$

Here, $\boldsymbol{H}$ is the lower triangular matrix that leads the algorithm to a sequential element-wise computation. Now to see the computational advantage, (19) can be extended to:

$$
\begin{aligned}
& \left(h_{11} u_{1}-y_{c(1)}\right)^{2}+\left(h_{21} u_{1}+h_{22} u_{2}-y_{c(2)}\right)^{2} \\
& +\left(h_{31} u_{1}+h_{32} u_{2}+h_{33} u_{3}-y_{c(3)}\right)^{2} \leqslant \rho^{2}(k),
\end{aligned}
$$

where $h_{i j}$ is the $(i, j)^{t h}$-element of $\boldsymbol{H}, u_{i}$ stands for the $i^{\text {th }}$-element of $\boldsymbol{u}$ and $y_{c(i)}$ refers to the $i^{t h}$-element of $\boldsymbol{y}_{\boldsymbol{c}}$. Based on (20), it begins computing the squared distance for the 1 st element $\left(u_{1}\right)$ up to the last element $\left(u_{3}\right)$ of $\boldsymbol{u}(k)$. Calculations for each element of $\boldsymbol{u}(k)$ initiate from $-\eta$ and ends at $\eta$. If the computed distance $d^{\prime}$ for $u_{i}$ is larger than $\rho^{2}$, then the algorithm discards the rest of the computations for the remaining elements. However, if $d^{\prime}$ for any element is smaller than $\rho^{2}$, then the SDA continues with the next elements. In this way, when $d^{\prime}$ for the final element $\left(u_{3}\right)$ is equal or smaller than $\rho^{2}$, then the corresponding $\boldsymbol{u}(k)$ is a prospective solution. Whenever we found a better prospective solution, the algorithm updates $\boldsymbol{u}^{\boldsymbol{o p}}$ and $\rho^{2}$ with $d^{\prime}$ as well. 


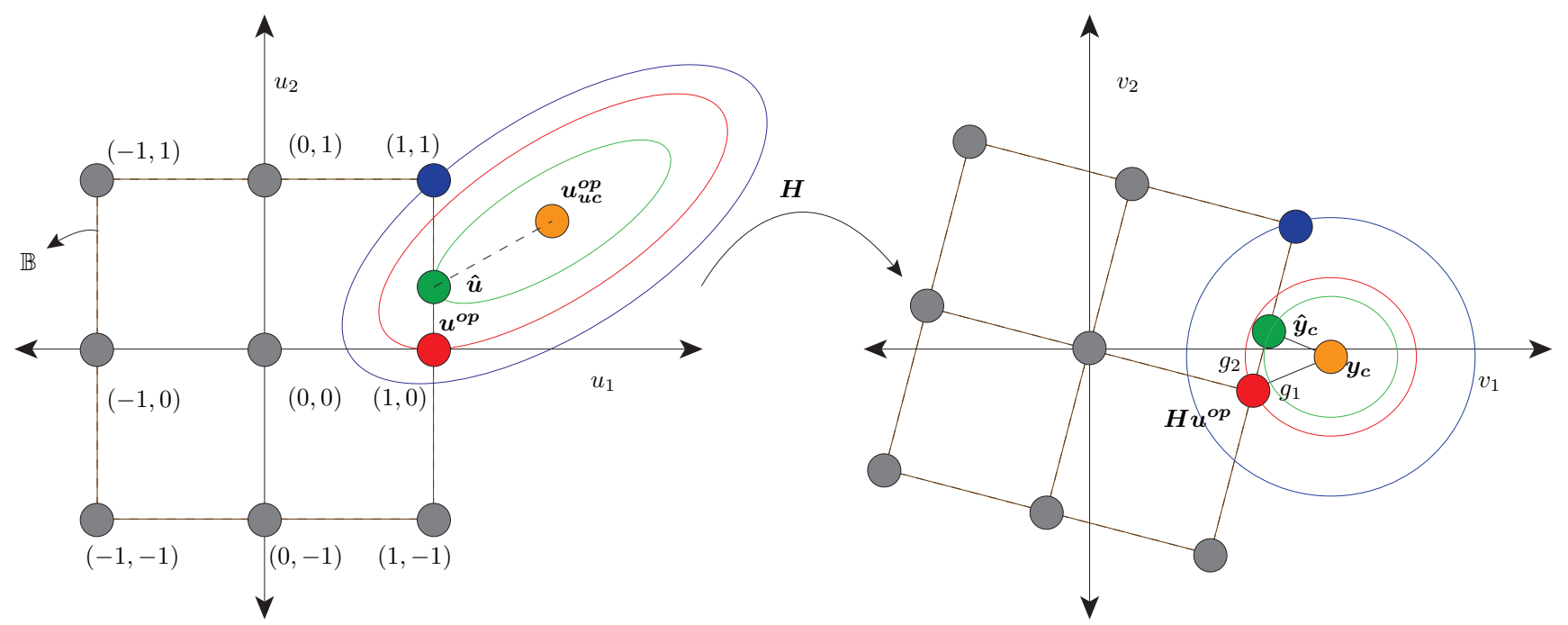

Fig. 3. The principle of the FCS-MPC with the modified sphere decoding algorithm in the original space- $u_{1} u_{2}$ and in the transformed space- $v_{1} v_{2}$.

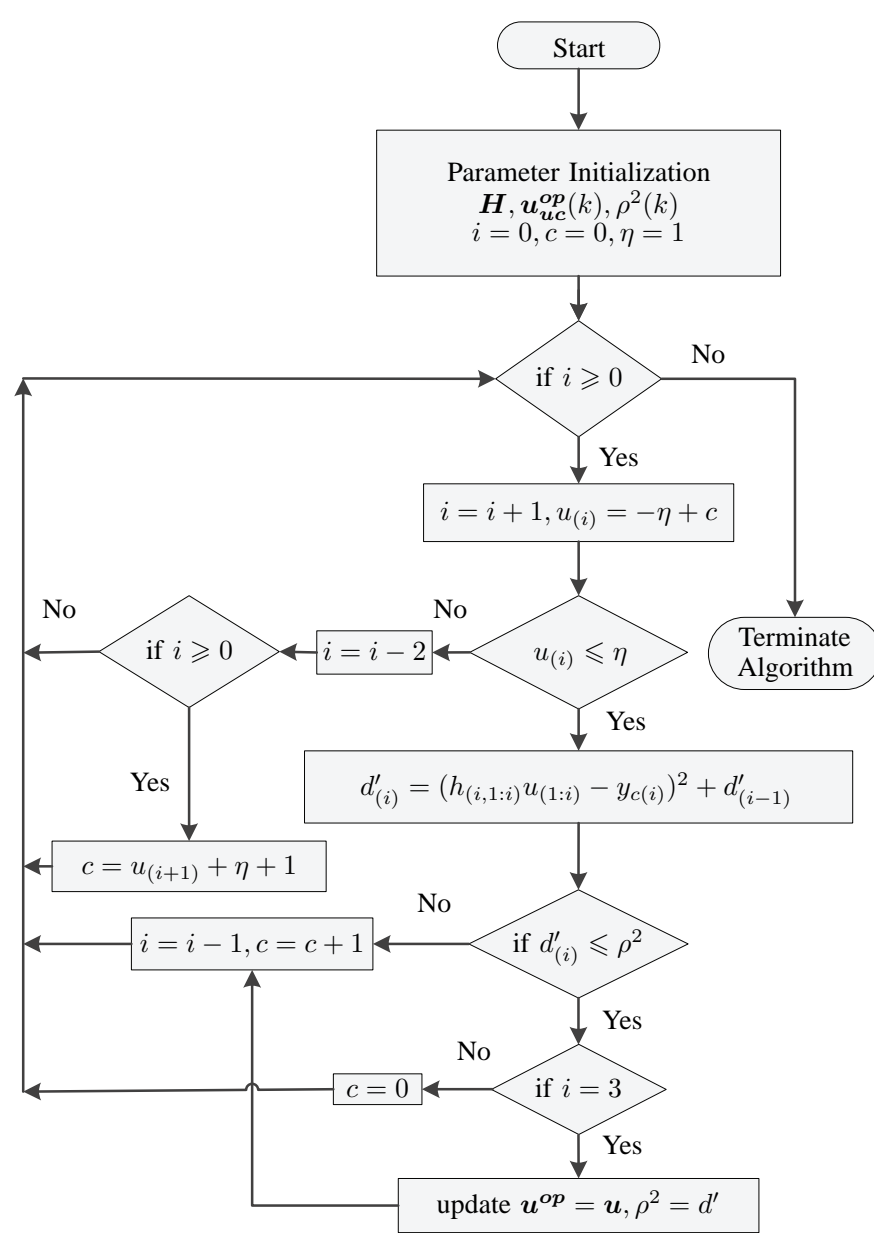

Fig. 4. Flow diagram of the sphere decoding algorithm for horizon one
Hence, the radius of the sphere is reduced if possible [20]; i.e., the search space is reduced. This approach can reduce the computational effort significantly.

With effective selection of the initial radius, the SDA can be extended to long horizons and the larger number of phase voltage level typically interested to multilevel converters.

\section{ENHANCING SDA FOR TRANSIENT OPERATIONS}

In this section, we illustrate the proposed modification to the SDA to achieve a fast optimization during transients of the power converter. This adaptation is based on the standard quadratic programming imposed on the integer quadratic problem (14).

To ease the understanding, we present the idea graphically in a two-dimensional space for the 3 -level HB converter (i.e., $\left.u_{1}, u_{2} \in\{-1,0,1\}\right)$ and horizon length $N=1$, see Fig. 3 . Thus, the finite set $\mathbb{U}$ has $3^{2}=9$ combinations for the control input $u$. At first, we define the convex hull (i.e., the envelope) of the lattice formed by the finite control set $\mathbb{U}$.

$$
\mathbb{B}=\text { ConvexHull }(\mathbb{U}), \mathbb{U} \subset \mathbb{B} .
$$

In general, during steady-state operation, the unconstrained optimal solution, $\boldsymbol{u}_{\boldsymbol{u c}}^{\boldsymbol{o p}}$ lies inside the boundary $\mathbb{B}$ (i.e., $\boldsymbol{u}_{\boldsymbol{u c}}^{\boldsymbol{o p}} \in$ $\mathbb{B})$. However, during transients (reference changes), $\boldsymbol{u}_{\boldsymbol{u}}^{o p}$ can be located far away from the lattice boundary. This results in a large initial sphere and, thereby, high computational time to find the optimal solution.

In dealing with this situation, whenever the unconstrained optimal solution, $\boldsymbol{u}_{u c}^{o p}$, lies outside of $\mathbb{B}$ (i.e., $u_{u c}^{o p} \notin \mathbb{B}$ ), we propose to project $u_{u c}^{o p}$ onto $\mathbb{B}$ by solving the following quadratic problem:

$$
\hat{\boldsymbol{u}}(k)=\arg \min _{\boldsymbol{u} \in \mathbb{B}}\left\{u^{T} W u+2 u^{T} \boldsymbol{F}(k)\right\} .
$$

where

$$
\hat{\boldsymbol{u}}(k) \in \mathbb{R}^{3 N} \in \mathbb{B} .
$$


We replicate this case in the first plot of Fig. 3, where the unconstrained optimal solution $\boldsymbol{u}_{\boldsymbol{u c}}^{\boldsymbol{o p}}$ (yellow point), is outside the boundary $\mathbb{B}$ and the optimal solution $\boldsymbol{u}^{\boldsymbol{o p}}$ (red point) to be found is on $\mathbb{B}$. As per definition in [23], the cost function $J(k)$ forms different ellipses (all points that lead to the same cost) with their centers at $\boldsymbol{u}_{\boldsymbol{u c}}^{\boldsymbol{o p}}$. The directions of an ellipse depend on the eigenvalues of $\boldsymbol{W}$. It is clear that ellipses closer to the center produce smaller cost values. Therefore, for the example in Fig. 3, the red point produces a lower cost than the blue one. Now, according to the quadratic programming problem (22), we obtain $\hat{\boldsymbol{u}}$ (green point) by projecting $\boldsymbol{u}_{\boldsymbol{u c}}^{\text {op }}$ onto the boundary. In this work, we propose to consider this projection as the new center in the original space $u_{1} u_{2}$ to run the SDA.

Therefore, instead of solving (18), during the transient we propose to solve the following optimization problem:

$$
\boldsymbol{u}^{\boldsymbol{o p}}(k)=\arg \left\{\min _{\boldsymbol{u} \in \mathbb{U}}\left\|\boldsymbol{H} \boldsymbol{u}(k)-\hat{\boldsymbol{y}}_{\boldsymbol{c}}(k)\right\|_{2}^{2}\right\} .
$$

where

$$
\hat{\boldsymbol{y}}_{\boldsymbol{c}}(\boldsymbol{k})=\boldsymbol{H} \hat{\boldsymbol{u}}(k) .
$$

It is important to emphasize that if the optimal solution $\boldsymbol{u}^{\boldsymbol{o p}}$ is one of the outer points of the original lattice formed by the finite control set, then solving (24) is, thus, equivalent to solve (18), whenever the unconstrained optimal solution is outside from the lattice boundary $\mathbb{B}$.

With the transformation matrix $\boldsymbol{H}$, the original space $u_{1} u_{2}$ is transformed into the new space $v_{1} v_{2}$. Here, the optimal solution is now the one, which is nearest neighbour to the center. This is because, the cost function forms now different circles (spheres for more than 2-dimension) with the defined center in the quantization problem. In essence, (22) brings the center of the sphere, $\boldsymbol{y}_{\boldsymbol{c}}(k)$ in (18), close to the lattice in an optimal manner by obtaining $\hat{\boldsymbol{y}}_{\boldsymbol{c}}(k)$. Here, $\boldsymbol{y}_{\boldsymbol{c}}$ (yellow point) and $\hat{\boldsymbol{y}}_{c}$ (green point) are the centers for the standard and the modified SDA respectively. It is clear from the figure that, using both $\boldsymbol{y}_{c}$ and $\hat{\boldsymbol{y}}_{\boldsymbol{c}}$ give us the same optimal solution $\boldsymbol{u}^{\boldsymbol{o p}}$, since from both, $\boldsymbol{H} \boldsymbol{u}^{o p}$ (red point) is the closest solution in the set. However, the target is closer to $\hat{\boldsymbol{y}}_{\boldsymbol{c}}$ than $\boldsymbol{y}_{\boldsymbol{c}}$, as $g_{2}<g_{1}$. Here, $g_{1}$ and $g_{2}$ are the distance from the target $\left(\boldsymbol{H} \boldsymbol{u}^{\boldsymbol{o p}}\right)$ to the centers $\boldsymbol{y}_{\boldsymbol{c}}$ and $\hat{\boldsymbol{y}}_{\boldsymbol{c}}$ respectively.

Consequently, whenever $\boldsymbol{y}_{\boldsymbol{c}}(k)$ is outside the ConvexHull, a new initial sphere with radius $\left\|\boldsymbol{H} \boldsymbol{u}(k-1)-\hat{\boldsymbol{y}}_{\boldsymbol{c}}(k)\right\|_{2}^{2}$ is setup, which is smaller than $\left\|\boldsymbol{H} \boldsymbol{u}(k-1)-\boldsymbol{y}_{\boldsymbol{c}}(k)\right\|_{2}^{2}$. This reduces the computational effort required by the SDA to obtain the optimal solution. Therefore, this modification helps to speedup the SDA during transients.

\section{Performance Evaluation: Simulation Results}

The simulation results of the FCS-MPC governing a threelevel converter using the improved SDA are carried out in this section. The system model uses the main parameters as shown in Table. I. The effectiveness of the proposal is verified by comparing it with the standard SDA used in the recent works [20], [22].

The results have been obtained in the, MATLAB-Simulink platform incorporated with PLECS. In Matlab, we use the
Table I

SYSTEM PARAMETERS

\begin{tabular}{ccc}
\hline \hline Variable & Description & Values \\
\hline$V_{d c}$ & dc voltage supply per HB & $180 \mathrm{~V}$ \\
$I^{\star}$ & Reference current amplitude & $4 \mathrm{~A}$ \\
$f$ & Fundamental frequency of the load current & $50 \mathrm{~Hz}$ \\
$R$ & Load resistor & $47 \Omega$ \\
$L$ & Load inductor & $15 \mathrm{mH}$ \\
$f_{s}$ & Sampling frequency & $10 \mathrm{kHz}$ \\
$\lambda$ & Weighting factor & 0.1 \\
$N$ & Horizon length & 5 \\
\hline \hline
\end{tabular}

following quadprog function, to find $\hat{\boldsymbol{u}}(k)$ in (22), with the constraints $l b=-\eta * \operatorname{ones}(p, 1)$ and $u b=\eta * \operatorname{ones}(p, 1)$. $\hat{\boldsymbol{u}}(\mathrm{k})=\mathbf{q u a d p r o g}(W, \boldsymbol{F}(k),[],[],[],[], l b, u b)$. Here, $l b$ and $u b$ are used to define the lower and upper boundary of the ConvexHull $\mathbb{B}$. Note that this is a simple quadratic programing problem since only lower and upper bounds are required as constraints.

Since, the steady-state performances are not the concern of this work, we have shown the results only for the transientstate. The simulations are performed for $30 \mathrm{~ms}$ and a step change $(-4 \mathrm{~A}$ to $4 \mathrm{~A})$ in the current reference is enforced at $20 \mathrm{~ms}$.

Figure (5) represents the results when the FCS-MPC uses the standard SDA. One can clearly see from Fig. (5e) that, during transients the algorithm requires extensively high computational time $\left(T_{c} \approx 3.5 \mathrm{~s}\right)$ compared with the steady-state to obtain the optimal solution. The average computational time, $T_{c}$ for steady-state is $1.2 \mathrm{~ms}$.

On the contrary, the system performances for the proposed SDA used in FCS-MPC are depicted in Figure. 6. Here, the computational time $T_{c}$ required in during transients is similar to the one needed in the steady-state; see Fig. (6e). The required computational time, $T_{c}$, for the proposed SDA during the whole simulation period is $1.2 \mathrm{~ms}$.

Comparing the resulting inverter voltages and currents obtained by both approaches, it can be clearly observed that the same optimal solution is obtained by both approaches. Nevertheless, the proposed SDA is able to find the optimal solutions efficiently in both steady-state and transients operation condition of the power converter.

\section{CONCLUSIONS}

This work proposed an improved SDA to solve the optimal control problem associated with FCS-MPC. The proposed approach is able to achieve the equivalent performance of standard SDA, in terms of the optimal solution. Consequently, the predictive controller ensures to obtain the optimal solution in transient-state, while maintaining the same computational effort like steady-state. 
(a)

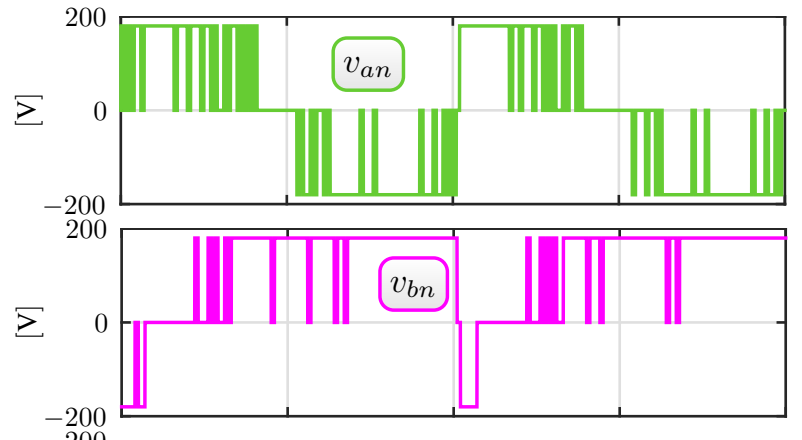

(c)

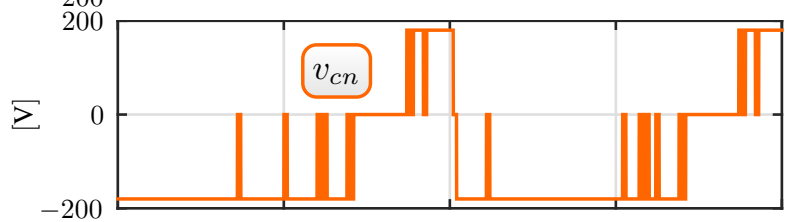

(d)

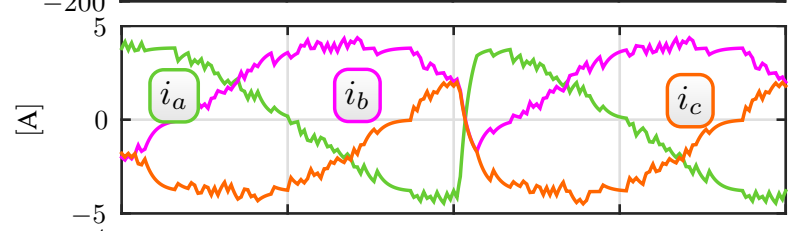

(e)

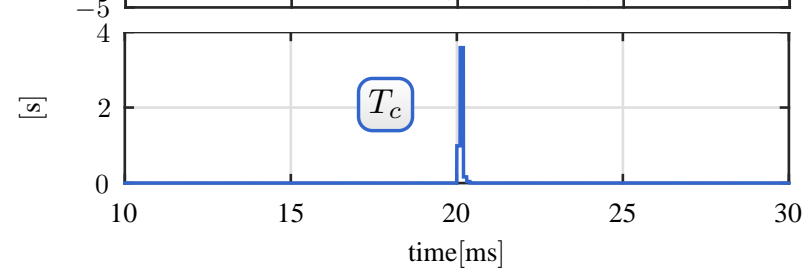

Fig. 5. The performance of the FCS-MPC strategy with the standard Sphere decoding algorithm (SDA) for a step change in the current references. Inverter voltages for, (a) Phase $a$, (b) Phase $b$, (c) Phase $c$. (d) Load currents, and (e) computational time.

The key novelty of the proposal lies in the way the center of the SDA is modified during transients. To do this, the unconstrained optimal solution is projected over the envelope of the original finite control set. Thus, a new center for the SDA is introduced. This speedup the optimization process since a smaller initial radius is obtained. As evidenced by the simulation results, the proposed SDA is able to quickly compute the optimal solution for the long-horizon direct MPC during both steady-state and transient operations of the power converter. Motivated by the present outcomes, future work will be focused on the experimental validation of this novelty and further contributions to make the SDA even faster.

\section{REFERENCES}

[1] S. Kouro, M. Malinowski, K. Gopakumar, J. Pou, L. Franquelo, B. Wu, J. Rodríguez, M. A. Pérez, and J. Leon, "Recent Advances and Industrial Applications of Multilevel Converters," IEEE Transactions on Industrial Electronics, vol. 57, no. 8, pp. 2553-2580, 2010.

[2] J. Rodriguez, L. Franquelo, S. Kouro, J. Leon, R. Portillo, M. Prats, and M. Perez, "Multilevel converters: An enabling technology for highpower applications," Proceedings of the IEEE, vol. 97, no. 11, pp. 17861817, Nov 2009.

[3] E. Al-Nabi, B. Wu, N. R. Zargari, and V. Sood, "Input power factor compensation for high-power csc fed pmsm drive using $\mathrm{d}$-axis stator current control," IEEE Transactions on Industrial Electronics, vol. 59, no. 2, pp. 752-761, Feb 2012.

[4] P. Acua, L. Morn, M. Rivera, R. Aguilera, R. Burgos, and V. G. Agelidis, "A single-objective predictive control method for a multivariable single-phase three-level npc converter-based active power filter," IEEE (a)

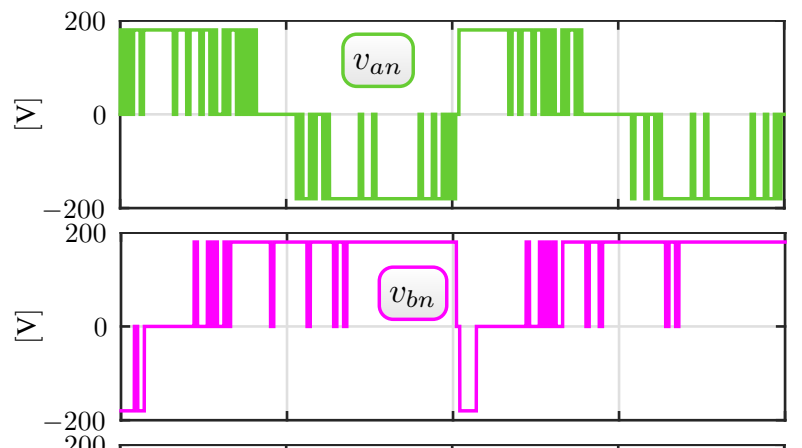

(c)

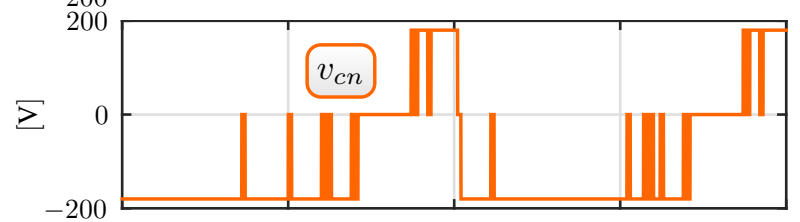

(d)

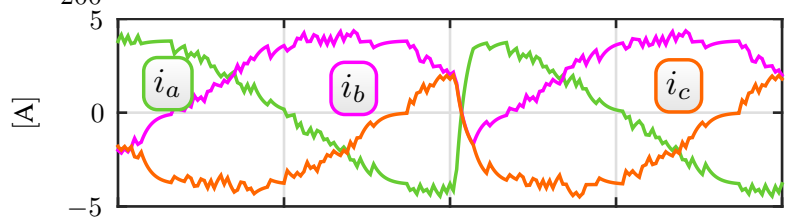

(e)

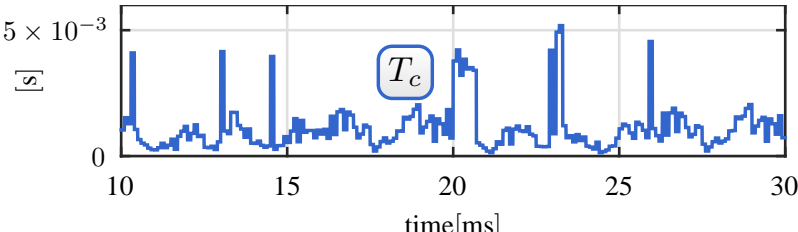

Fig. 6. The performance of the FCS-MPC strategy with the proposed Sphere decoding algorithm (SDA) for a step change in the current references. Inverter voltages for, (a) Phase $a$, (b) Phase $b$, (c) Phase $c$. (d) Load currents, and (e) computational time.

Transactions on Industrial Electronics, vol. 62, no. 7, pp. 4598-4607, July 2015.

[5] M. R. Islam, Y. Guo, and J. Zhu, "A high-frequency link multilevel cascaded medium-voltage converter for direct grid integration of renewable energy systems," IEEE Transactions on Power Electronics, vol. 29, no. 8, pp. 4167-4182, Aug 2014.

[6] L. Popova, J. Pyrhonen, K. L. Ma, and F. Blaabjerg, "Device loading of modular multilevel converter mmc in wind power application," in Power Electronics Conference (IPEC-Hiroshima 2014 - ECCE-ASIA), 2014 International, May 2014, pp. 548-554.

[7] S. Vazquez, J. Leon, L. Franquelo, J. Rodriguez, H. Young, A. Marquez, and P. Zanchetta, "Model predictive control: A review of its applications in power electronics," IEEE Ind. Electron. Mag., vol. 8, no. 1, pp. 16-31, March 2014.

[8] P. Lezana, R. Aguilera, and D. Quevedo, "Model predictive control of an asymmetric flying capacitor converter," Industrial Electronics, IEEE Transactions on, vol. 56, no. 6, pp. 1839-1846, June 2009.

[9] T. Geyer, G. Papafotiou, and M. Morari, "Model Predictive Direct Torque Control-Part I: Concept, Algorithm, and Analysis," IEEE Transactions on Industrial Electronics, vol. 56, no. 6, pp. 1894-1905, 2009.

[10] G. Papafotiou, J. Kley, K. G. Papadopoulos, P. Bohren, and M. Morari, "Model predictive direct torque control-Part II: Implementation and experimental evaluation," IEEE Transactions on Industrial Electronics, vol. 56, no. 6, pp. 1906-1915, Jun. 2009.

[11] N. Oikonomou, C. Gutscher, P. Karamanakos, F. D. Kieferndorf, and T. Geyer, "Model Predictive Pulse Pattern Control for the Five-Level Active Neutral-Point-Clamped Inverter," IEEE Transactions on Industry Applications, vol. 49, no. 6, pp. 2583-2592, 2013.

[12] R. P. Aguilera, P. Lezana, and D. E. Quevedo, "Switched Model Predictive Control for Improved Transient and Steady-State Performance," IEEE Transactions on Industrial Informatics, vol. 11, no. 4, pp. 968977, Aug. 2015. 
[13] R. P. Aguilera, P. Lezana, G. Konstantinou, P. Acuna, B. Wu, S. Bernet, and V. G. Agelidis, "Closed-loop SHE-PWM technique for power converters through Model Predictive Control," in Industrial Electronics Society, IECON 2015 - 41st Annual Conference of the IEEE, Yokohama, Nov. 2015, pp. 005 261-005 266.

[14] J. Rodríguez, M. P. Kazmierkowski, J. Espinoza, P. Zanchetta, H. AbuRub, H. A. Young, and C. A. Rojas, "State of the Art of Finite Control Set Model Predictive Control in Power Electronics," IEEE Transactions on Industrial Informatics, vol. 9, no. 2, pp. 1003-1016, May 2013.

[15] S. Vazquez, A. Marquez, R. Aguilera, D. Quevedo, J. I. Leon, and L. G. Franquelo, "Predictive optimal switching sequence direct power control for grid-connected power converters," IEEE Transactions on Industrial Electronics, vol. 62, no. 4, pp. 2010-2020, April 2015.

[16] T. Geyer, "A Comparison of Control and Modulation Schemes for Medium-Voltage Drives: Emerging Predictive Control Concepts Versus PWM-Based Schemes," IEEE Transactions on Industry Applications, vol. 47, no. 3, pp. 1380-1389, 2011.

[17] P. Karamanakos, T. Geyer, N. Oikonomou, F. D. Kieferndorf, and S. Manias, "Direct Model Predictive Control: A Review of Strategies That Achieve Long Prediction Intervals for Power Electronics," IEEE Industrial Electronics Magazine, vol. 8, no. 1, pp. 32-43, Mar. 2014.

[18] C. Buccella, C. Cecati, and H. Latafat, "Digital control of power converters - a survey," Industrial Informatics, IEEE Transactions on, vol. 8, no. 3, pp. 437-447, Aug 2012.

[19] T. Vyncke, S. Thielemans, and J. Melkebeek, "Finite-set model-based predictive control for flying-capacitor converters: Cost function design and efficient fpga implementation," Industrial Informatics, IEEE Transactions on, vol. 9, no. 2, pp. 1113-1121, May 2013.

[20] T. Geyer and D. E. Quevedo, "Multistep Finite Control Set Model Predictive Control for Power Electronics," IEEE Transactions on Power Electronics, vol. 29, no. 12, pp. 6836-6846, 2014.

[21] — "Performance of Multistep Finite Control Set Model Predictive Control for Power Electronics," IEEE Transactions on Power Electronics, vol. 30, no. 3, pp. 1633-1644, Mar. 2015.

[22] R. P. Aguilera, R. Baidya, P. Acuna, S. Vazquez, T. Mouton, and V. G. Agelidis, "Model predictive control of cascaded h-bridge inverters based on a fast-optimization algorithm," in Industrial Electronics Society, IECON 2015 - 41st Annual Conference of the IEEE, Nov 2015, pp. 004 003-004 008.

[23] D. E. Quevedo, R. P. Aguilera, and T. Geyer, "Predictive Control in Power Electronics and Drives: Basic Concepts, Theory, and Methods," in Studies in Computational Intelligence. Cham: Springer International Publishing, Jan. 2014, pp. 181-226.

[24] R. A. Horn and C. R. Johnson., "Matrix Analysis," Cambridge University Press, Cambridge, UK., 1985. 